\title{
AG Draconis observed with XMM-Newton
}

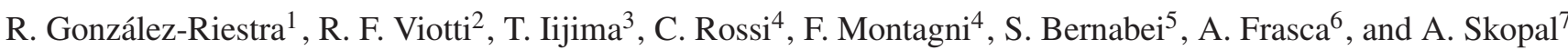 \\ 1 XMM Science Operations Centre, ESAC, PO Box 78, 28691 Villanueva de la Cañada, Madrid, Spain \\ e-mail: Rosario.Gonzalez@sciops.esa.int \\ 2 INAF, Istituto di Astrofisica Spaziale e Fisica Cosmica di Roma, via del Fosso del Cavaliere 100, 00133 Roma, Italy \\ 3 INAF - Osservatorio Astronomico di Padova, Sezione di Asiago, 36012 Asiago, Italy \\ 4 Dipartimento di Fisica, Università La Sapienza, Piazzale Aldo Moro 3, 00185 Roma, Italy \\ 5 INAF - Osservatorio Astronomico di Bologna, via Ranzani 1, 40127 Bologna, Italy \\ ${ }^{6}$ INAF - Osservatorio Astrofisico di Catania, via S. Sofia 78, 95123 Catania, Italy \\ 7 Astronomical Institute, Slovak Academy of Sciences, 05960 Tatranská Lomnica, Slovakia
}

Received 31 August 2007 / Accepted 14 January 2008

\section{ABSTRACT}

\begin{abstract}
Context. AG Draconis is the brightest symbiotic star in X-rays and one of the prototypes of the supersoft X-ray source class. Aims. Study of the X-ray spectrum of this peculiar binary system, covering both quiescence and activity periods, is necessary to investigate the physics of the high temperature spectral component, and to unveil the origin of the outbursts.

Methods. X-ray and UV observations with XMM-Newton during 2003-2005 and coordinated optical spectrophotometric monitoring, together with archive data, are employed to derive the behaviour of the high energy source of the AG Dra system during different orbital and activity phases.

Results. During quiescence the X-ray emission is very soft and is close in strength to the previous ROSAT observations, with an estimated luminosity of $2600 L_{\odot}$ and a radius of $0.06 R_{\odot}$. We also found a 20\% flux decrease in June 2005 at the time when a $U$-band minimum coincided with a $V$-band maximum. The X-ray flux in the XMM range largely decreases, and even vanishes near the optical light maxima (bursts). The UV fluxes measured with the XMM Optical Monitor is close to the fluxes observed with the IUE satellite. There is a marked anticorrelation between X-ray fluxes, and ultraviolet and optical fluxes, indicating that during outburst the WD is mostly emitting below $0.1 \mathrm{keV}$. Conversely, the large strengthening of the He II $4686 \AA$ emission during the October 2003 and July 2005 light maxima indicates a marked increase of the far-UV brightness especially during the early stages of the outbursts. A high energy $0.5-0.6 \mathrm{keV} \mathrm{X}$-ray excess seems to be present both in quiescence and outburst.

Conclusions. This is the first time that X-ray spectra of AG Draconis during an active phase are obtained. These data have allowed us to investigate the change of the energy distribution. The anti-correlation between X-ray and optical/ultraviolet emission appears to be a general feature of AG Dra independent of the type and strength of the outburst. We suggest that during outburst the WD radiation increases, but is strongly absorbed by the circumstellar ionised gas. The newly-found high energy X-ray component is probably an indication of the presence of a shocked region produced by interaction of the WD wind with the circumbinary envelope fed by the red giant's wind.
\end{abstract}

Key words. X-rays: individuals: AG Draconis - X-rays: binaries

\section{Introduction}

Prior to the ROSAT era, very few symbiotic systems had been detected in X-rays, and therefore our understanding of the origin of their emission and of their variations during the different activity phases was incomplete. The large number of X-ray spectra of symbiotic stars obtained with ROSAT allowed to classify them into three basic categories: supersoft spectra, spectra peaking at approximately $0.8 \mathrm{keV}$, and hard X-ray sources. These three categories were tentatively identified with hot White Dwarfs (WD), hot shocked gas, and systems with a neutron star, respectively (Mürset et al. 1997). AG Draconis was the prototype of the first of these three classes. With the wealth of data provided by the new high energy experiments, the overall picture becomes more complicated, as there exist systems in which the supersoft component dominates, but that also have a substantial hard emission (e.g. CH Cyg, Mukai et al. 2007). Therefore, these three classes established by ROSAT do not mutually exclude each other.

AG Draconis, being the brightest symbiotic system in X-rays and one of the prototypes of the "Super Soft X-ray Sources" class, is one of the best monitored symbiotic systems and one of the preferred targets for multiwavelength studies.

AG Dra is a halo binary system composed of a metal-poor $\mathrm{K}$ giant and a hot WD. It is at high-latitude $\left(b_{\mathrm{II}}=+41^{\circ}\right)$, and has a low reddening $\left(N_{\mathrm{H}}=3.15 \times 10^{20} \mathrm{~cm}^{-2}\right.$, Viotti et al. 1983). The orbital period of 554 days is clearly seen in the $U$-band (Meinunger 1979). In the following we make use of the orbital phases computed according to the ephemeris:

$U_{\min }=2443629.17+549.73 \times E$

(Gális et al. 1999; see also Skopal et al. 2004). A second period with a time scale of about $350 \mathrm{~d}$ is seen during quiescence in the $B$ and $V$ bands, and in the radial velocity curve as well, which could be associated with pulsations of the cool component (e.g. Gális et al. 1999).

The behaviour of the system is characterised by long periods of quiescence $(V \sim 9.9)$ that can last more than one decade and phases of activity composed of several outbursts. AG Dra has undergone several phases of activity in the last quarter of century: 1980-82 (two outbursts in November 1980 


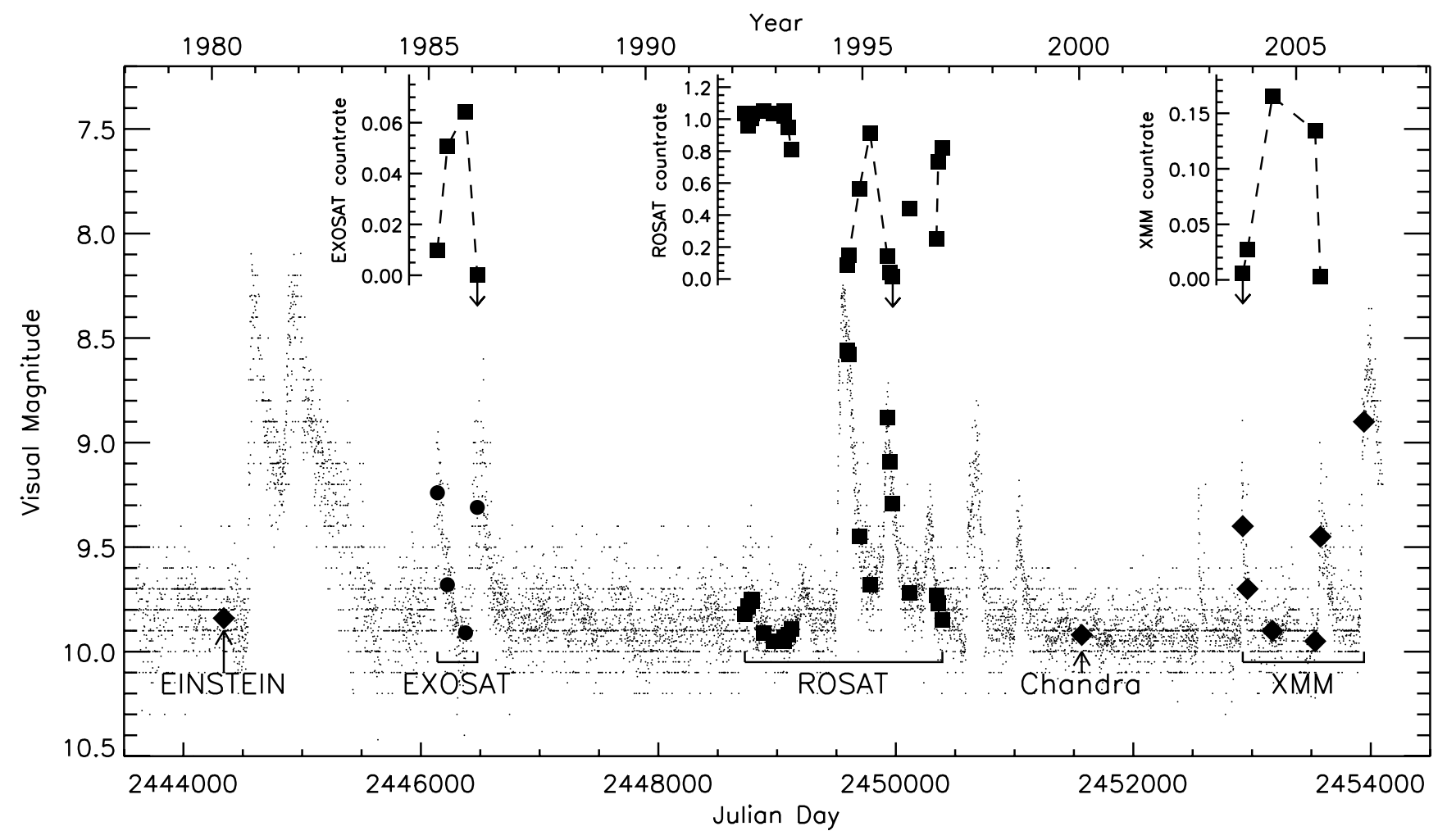

Fig. 1. Comparison of the AAVSO optical light curve of AG Dra (dots) with the X-ray light curves (top of the figure). The solid points superposed on the light curve mark the visual magnitude at the times of the X-ray observations. The plots in the upper part of the figure show the EXOSAT, ROSAT and XMM light curves (units are counts per sec).

and August 1981, and possibly a third one at the beginning of 1983), 1985-86 (two outbursts in March 1985 and January 1986), and 1994-98 (five outbursts) (e.g. González-Riestra et al. 1999; Viotti et al. 2003). The last activity phase had three minor outbursts in October 2001, September 2002, September 2003, and July 2005, and a bright one that started in July 2006. González-Riestra et al. (1999), in their analysis of the behaviour of the He II $1640 \AA$ emission line and of the far-UV continuum, identified two types of outbursts, cool or hot depending on whether the WD temperature at maximum luminosity is lower or higher than during quiescence. The strong outbursts of 1981 and 1994 were "cool", while the minor 1985 and 1986 outbursts were "hot".

The September 2003 activity phase reached a maximum visual magnitude of 9.2 at the beginning of October (Skopal et al. 2004). The outburst lasted approximately two months. The June 2005 outburst was of similar brightness, but longer. The June 2006 outburst was the brightest one in the last ten years, with a visual magnitude at maximum of $V \sim 8.5$, close to that reached in 1994 and 1980-81. In August 2007 the system was still at visual magnitude $\sim 9.4$.

The separation between the two last pairs of maxima was approximately one year, similar to the second period found in the analysis of the light curve in quiescence ( 378 days according to Bastian 1998). This was also the case after the June 1994 large outburst, when four secondary maxima took place with intervals of about one year.

Our group has extensively observed AG Dra in the optical, $\mathrm{UV}$ and X-ray ranges along all these phases. The analysis of these observations have been published in Viotti et al. (1983), Viotti et al. (1984), Greiner et al. (1997) and González-Riestra et al. (1999).

\subsection{The $X$-ray history of $A G$ Draconis}

AG Draconis has been observed in X-rays for more than 25 years. Figure 1 illustrates the AAVSO visual light curve of AG Dra and marks the times of the X-ray observations. The EXOSAT, ROSAT and XMM-Newton count rates are also shown.

The X-ray emission from AG Dra was first detected by the Einstein Observatory just before the 1980 outburst at a countrate of 0.27 IPC cts/s (Anderson et al. 1981). These observations were consistent with a blackbody spectrum with $T=186000 \mathrm{~K}$ (Kenyon 1988). Anderson et al. (1981) suggested the existence of an additional bremsstrahlung source $(k T=0.1 \mathrm{keV})$.

The 1985-86 weak outbursts were monitored by EXOSAT (Viotti et al. 1995). Two observations were obtained near the optical peaks and two others in the period of visual minimum in between. The first observation, shortly after the discovery of the outburst, gave a low count in the thin Lexan filter (see Fig. 1). In June 1985, when the system was close to its visual minimum, the observed countrate was a factor of 5 higher and a comparable value was obtained in November. The source was not detected in the last pointing $\left(<0.003 \mathrm{cts} \mathrm{s}^{-1}\right)$, at the time of the January 1986 optical maximum. Piro et al. (1985) estimated a blackbody temperature of $200000 \mathrm{~K}$ (i.e., $k T \simeq 17 \mathrm{eV}$ ) in the inter-outburst period.

The AG Dra region was scanned for 10 days during the ROSAT All-Sky-Survey, and subsequently, several pointed observations were performed with the PSPC in 1992-93. The rest of the observations were performed with the HRI, except for one in September 1994 when the PSPC and the Boron filter were used. The ROSAT observations of AG Dra until February 1996 were analysed by Greiner et al. (1997). The mean PSPC countrate during the All Sky Survey and the pointed observations 
Table 1. Summary of EPIC XMM-Newton observations of AG Draconis.

\begin{tabular}{lccccc}
\hline \hline Instrument & \multicolumn{1}{c}{ Mode } & Filter & Start time $($ UT) & Duration $/ T_{\text {exp }}(\mathrm{s})^{*}$ & Countrate $^{* *}$ \\
\hline October 10 2003 $(\Phi=0.91)$ & & & & \\
\hline EPIC-pn & Small window & Medium & $01: 55$ & $24880 / 17121$ & $(-5.42 \pm 1.94) \times 10^{-3}$ \\
EPIC-MOS1 & Small window & Medium & $00: 49$ & $24657 / 23918$ & $(-0.60 \pm 2.86) \times 10^{-4}$ \\
EPIC-MOS2 & Small window & Medium & $00: 49$ & $24662 / 23918$ & $(4.07 \pm 6.05) \times 10^{-4}$ \\
\hline November 19 $2003(\Phi=0.98)$ & & & & \\
\hline EPIC-pn & Large window & Thin & $01: 07$ & $10496 / 9217$ & $(2.76 \pm 0.21) \times 10^{-2}$ \\
EPIC-MOS1 & Large window & Thin & $00: 47$ & $11972 / 11065$ & $(1.44 \pm 0.49) \times 10^{-3}$ \\
EPIC-MOS2 & Small window & Thin & $00: 47$ & $11962 / 11562$ & $(1.83 \pm 0.52) \times 10^{-3}$ \\
\hline June 15 2004 $(\Phi=0.36)$ & & & & \\
\hline EPIC-pn & Large window & Thin & $09: 13$ & $16896 / 12354$ & $(1.65 \pm 0.04) \times 10^{-1}$ \\
EPIC-MOS1 & Large window & Thin & $08: 53$ & $18372 / 14480$ & $(7.59 \pm 0.84) \times 10^{-3}$ \\
EPIC-MOS2 & Full frame & Thin & $08: 53$ & $18372 / 14354$ & $(3.51 \pm 0.62) \times 10^{-3}$ \\
\hline June 11 2005 $(\Phi=0.01)$ & & & & \\
\hline EPIC-pn & Large window & Thin & $10: 49$ & $19970 / 18306$ & $(1.34 \pm 0.03) \times 10^{-1}$ \\
EPIC-MOS1 & Large window & Thin & $10: 20$ & $21972 / 21590$ & $(1.98 \pm 0.50) \times 10^{-3}$ \\
EPIC-MOS2 & Full frame & Thin & $10: 21$ & $21972 / 21481$ & $(3.29 \pm 0.49) \times 10^{-3}$ \\
\hline July 25 2005 $(\Phi=0.09)$ & & & & \\
\hline EPIC-pn & Large window & Thin & $09: 43$ & $23606 / 11483$ & $(3.62 \pm 1.44) \times 10^{-3}$ \\
EPIC-MOS1 & Large window & Thin & $08: 14$ & $25608 / 15614$ & $(4.16 \pm 5.24) \times 10^{-4}$ \\
EPIC-MOS2 & Full frame & Thin & $08: 14$ & $25598 / 16318$ & $(1.14 \pm 0.46) \times 10^{-3}$ \\
\hline
\end{tabular}

Phases refer to the $U$ band light curve (see text).

${ }^{*} T_{\exp }$ is the effective exposure time after removal of background flares.

** EPIC countrates $(0.2-1 \mathrm{keV})$ in $\mathrm{cts} \mathrm{s}^{-1}$.

in quiescence was $0.99 \pm 0.07 \mathrm{cts} \mathrm{s}^{-1}$. The spectrum was in all cases well fitted by an absorbed blackbody spectrum. Fixing the column density $N_{\mathrm{H}}$ at its galactic value $\left(3.15 \times 10^{20} \mathrm{~cm}^{-2}\right)$ the temperature was between 162000 and $174000 \mathrm{~K}$. The luminosity of $10^{37} \mathrm{erg} \mathrm{s}^{-1}$ suggests hydrogen burning on the surface of a low mass $\left(<0.6 M_{\odot}\right)$ white dwarf. Here and in the following we shall assume for AG Dra the distance of $2.5 \mathrm{kpc}$ suggested by Mikolajewska et al. (1995). As previously observed with EXOSAT, the X-ray flux dropped substantially during the 1994 and 1995 optical outbursts, and nearly recovered the quiescence level in the period between the peaks. The lack of PSPC observations makes the analysis of the X-ray decrease during outburst difficult. According to Greiner et al. (1997) the low HRI countrate at the beginning of the outburst can be reproduced either by an increase in the absorbing column by a factor of three (with a constant blackbody temperature of $174000 \mathrm{~K}$ ) or by a decrease of the temperature from 164000 to $116000 \mathrm{~K}$. However, the enhanced absorption column can be discarded, since it would have been observed in the PSPC + Boron filter observation of September 1994. Three additional observations were performed by ROSAT-HRI in September, October and November 1996 (Catalogue of ROSAT HRI Pointed Observations, ROSAT News. 71, 2000). The general trend was confirmed: the X-ray source was fainter in the first one, taken just at the end of a small outburst, and then brightened when the optical flux was going back to the quiescence value (see Fig. 1). AG Draconis was also observed with Chandra-ACIS in January 2000, during a long period of quiescence.

\subsection{XMM observations}

AG Draconis has been observed observed by XMM-Newton (Jansen et al. 2001) six times. The first was on October 10, 2003, at the beginning of a minor outburst (observation Id. 0151530401, P.I. J. Sokoloski). New observations were taken a few weeks later on November 19, 2003 (observation Id. 0147131101), after the outburst maximum and when the system had faded to $V=9.58$ (Skopal et al. 2004). This and the following three observations were made under the approved proposal 014713, with González-Riestra as P.I. Then, the system was observed during two periods of quiescence: June 15, 2004 (observation Id. 0147130101) and June 11, 2005 (observation Id. 0147130901). This last observation was taken with the specific purpose of determining any X-ray modulation in coincidence with the $U$-band minimum, that is at conjunction of the binary system, near orbital phase zero. By chance this observation was made just before the start of a new outburst. The next observation (observation Id. 0147131001) was obtained when the system was in outburst, on July 25, 2005. A sixth observation (P.I. J. Sokoloski, observation Id. 0151530501) was made during the July 2006 outburst.

The system was observed by all the instruments on-board XMM-Newton, including the Optical Monitor (OM) for ultraviolet observations. The target was not detected in any exposure with the high resolution X-ray spectrograph (RGS) since, because of its very soft spectrum (see below), the source was too faint for the RGS spectral range. Details of the EPIC observations are summarised in Table 1. The X-ray and UV observations are presented in Sects. 2 and 3. Section 4 describes the coordinated optical spectroscopic and photometric observations. The results are discussed in Sect. 5, and conclusions are given in Sect. 6.

\section{The X-ray data}

The XMM-Newton data were reduced with the most recent version of the Science Analysis System (SAS 7.0), and with the most up-to-date calibration data as of September 2006. Some of the observations were affected by background flares. These periods of high radiation have been removed before analysing the data. Spectra from the three EPIC cameras have been extracted 

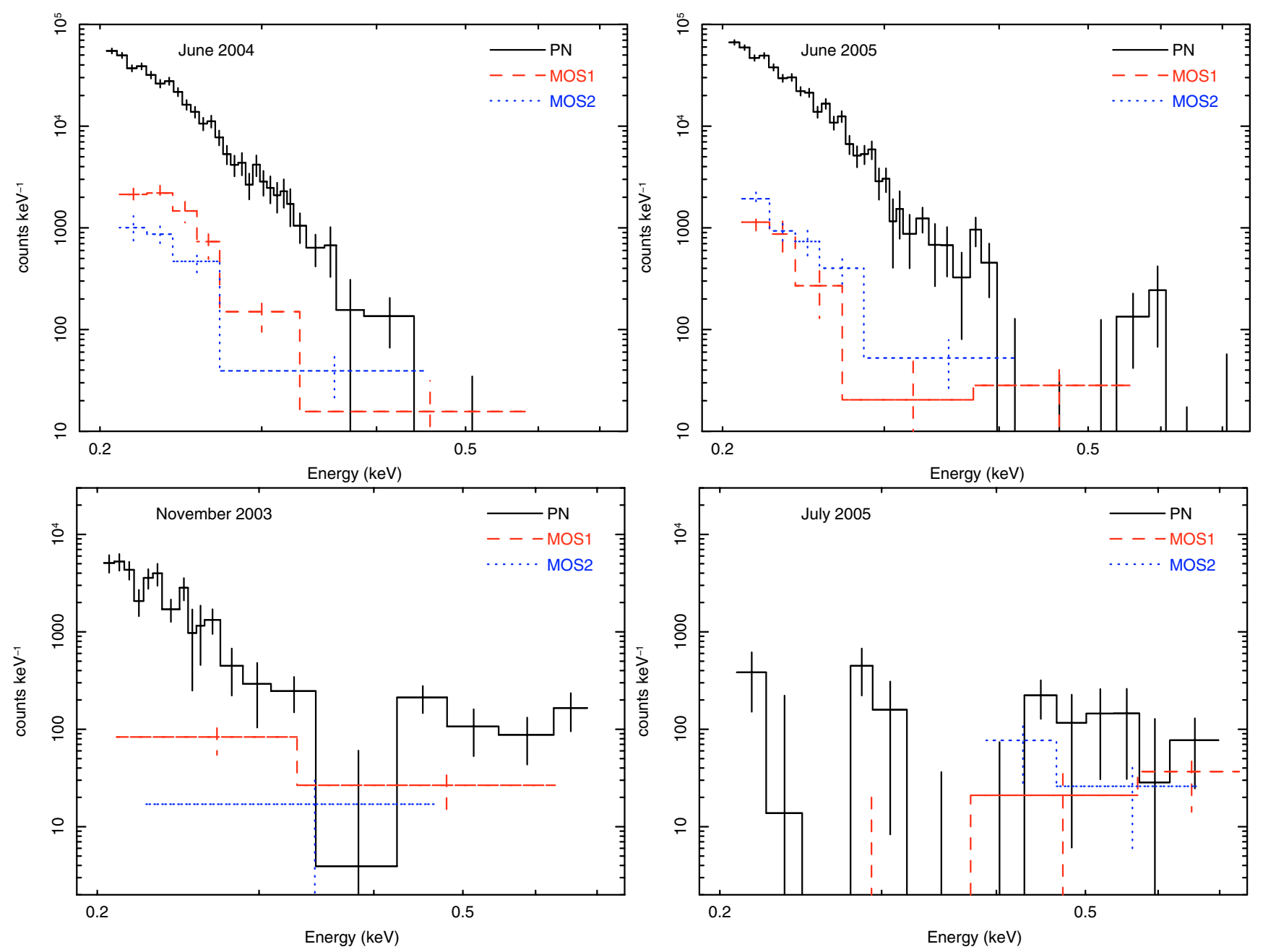

Fig. 2. XMM EPIC spectra of AG Draconis. The two top panels show the quiescence spectra of June 2004 (left) and June 2005 (right). Spectra in outburst are shown in the bottom panels: November 2003 (left), and July 2005 (right). The spectra shown in the figures have been rebinned to a minimum of 10 counts/channel for display purposes. The fits described in the text have been performed to the spectra rebinned so as to oversample the full width half maximum of the energy resolution by a factor 3 and to have a minimum of 20 counts per bin.

from a circular region with a radius of about 40 arcsec around the target. For EPIC-pn and EPIC-MOS in Full Frame or Large Window mode, the background was extracted from a nearby region, while for EPIC-MOS in Small Window mode, an annulus around the target was used instead.

Table 1 gives the EPIC count rate in the range $0.2-1.0 \mathrm{keV}$ for each observation. Rebinned spectra from the three EPIC cameras are shown in Fig. 2. Fits have been performed in all cases on spectra rebinned so as to oversample the full width half maximum of the energy resolution by a factor of 3 and to have a minimum of 20 counts per bin. Only data at energies higher than $200 \mathrm{eV}$ were used.

\subsection{The quiescence}

The X-ray spectrum of AG Draconis in quiescence is typical of a supersoft source (see Fig. 2). The spectra of June 2004 and June 2005 are very similar, the only difference being that in the latter the flux was slightly lower and there was an emission excess between 0.5 and $0.6 \mathrm{keV}$. We have fitted the EPIC spectra in the region $0.2-1 \mathrm{keV}$ with a blackbody affected by interstellar absorption. Unfortunately, the low-energy limit, set at $200 \mathrm{eV}$, and the strong interrelation of the hydrogen column density do not allow us to usefully constrain the fit parameters. We have fitted the data with the model derived from the ROSAT PSPC data (Greiner et al. 1997): a blackbody with $k T=14.5 \mathrm{eV}$ and the galactic hydrogen column density $\left(N_{\mathrm{H}}=3.15 \times 10^{20} \mathrm{~cm}^{-2}\right)$. The derived integrated unabsorbed blackbody flux are 1.52 and $1.22 \times 10^{-8} \mathrm{erg} \mathrm{s}^{-1} \mathrm{~cm}^{-2}$ in June 2004 and June 2005, respectively, corresponding to luminosities of 2960 and $2370 L_{\odot}$, for a distance of $2.5 \mathrm{kpc}$, in agreement with the mean value derived from the ROSAT quiescence observations $\left(2500 L_{\odot}\right)$. For these luminosities, the radius of the hot star would be 0.065 and $0.058 R_{\odot}$, respectively. Formally, the fits to the data of both dates (keeping the blackbody temperature fixed at $14.5 \mathrm{eV}$ ) are better with a lower hydrogen column density $\left(2.7 \times 10^{20} \mathrm{~cm}^{-2}\right)$, that would lead to luminosities and radii of $2220 L_{\odot}, 0.056 R_{\odot}$ (in 2004) and $1770 L_{\odot}, 0.050 R_{\odot}($ in 2005).

\subsection{The active epochs}

So far AG Draconis has been observed with XMM-Newton during three outbursts: in October and November 2003, in July 2005 and in July 2006 (this last one is not considered here as the XMM observations are not public at the time of writing). 
Table 2. Summary of XMM-Newton OM observations of AG Draconis.

\begin{tabular}{|c|c|c|c|c|}
\hline Filter & UT start & Duration (s) & Countrate & Flux \\
\hline \multicolumn{2}{|c|}{ October 102003} & Phase $0.91^{*}$ & & \\
\hline$U V W 2$ & $06: 42$ & 1200 & $104 \pm 2$ & $6.15 \pm 0.01$ \\
\hline$U V W 2$ & 07:07 & 1200 & $102 \pm 2$ & $6.20 \pm 0.04$ \\
\hline$U V W 2$ & 07:33 & 1200 & $101 \pm 2$ & $5.94 \pm 0.01$ \\
\hline$U V W 2$ & $07: 58$ & 1200 & $99 \pm 2$ & $5.99 \pm 0.04$ \\
\hline$U V W 2$ & $08: 24$ & 1200 & $95 \pm 2$ & $5.59 \pm 0.01$ \\
\hline \multicolumn{2}{|c|}{ November 192003} & Phase $0.98^{* *}$ & & \\
\hline UVW1 & $02: 59$ & 4400 & $493 \pm 10$ & $2.40 \pm 0.02$ \\
\hline$U V W 1$ & $04: 18$ & 4400 & $491 \pm 10$ & $2.42 \pm 0.01$ \\
\hline$U V W 1$ & $05: 36$ & 4400 & $497 \pm 10$ & $2.39 \pm 0.02$ \\
\hline$U V M 2$ & $06: 55$ & 4400 & $91 \pm 2$ & $2.05 \pm 0.01$ \\
\hline UVM2 & $08: 13$ & 4400 & $90 \pm 2$ & $2.02 \pm 0.01$ \\
\hline$U V W 2$ & 09:32 & 4400 & $36 \pm 1$ & $2.08 \pm 0.01$ \\
\hline$U V W 2$ & $10: 50$ & 4400 & $37 \pm 1$ & $2.11 \pm 0.01$ \\
\hline \multicolumn{2}{|c|}{ June 152004} & Phase 0.36 & & \\
\hline$U V W 1$ & 08:57 & 4400 & $348 \pm 5$ & $1.73 \pm 0.01$ \\
\hline UVM2 & $10: 16$ & 4400 & $60 \pm 3$ & $1.36 \pm 0.01$ \\
\hline$U V M 2$ & $11: 34$ & 2000 & $60 \pm 1$ & $1.35 \pm 0.01$ \\
\hline$U V W 2$ & $12: 43$ & 4400 & $23 \pm 1$ & $1.35 \pm 0.01$ \\
\hline \multicolumn{2}{|c|}{ June 112005} & Phase 0.01 & & \\
\hline UVM2 & $11: 25$ & 4400 & $53 \pm 1$ & $1.19 \pm 0.01$ \\
\hline UVM2 & $12: 14$ & 4400 & $53 \pm 1$ & $1.18 \pm 0.01$ \\
\hline UVM2 & $13: 32$ & 3300 & $53 \pm 1$ & $1.18 \pm 0.01$ \\
\hline UVM2 & $14: 32$ & 3600 & $53 \pm 1$ & $1.19 \pm 0.01$ \\
\hline UVM2 & $15: 38$ & 2880 & $53 \pm 1$ & $1.18 \pm 0.01$ \\
\hline \multicolumn{2}{|c|}{ July 252005} & Phase 0.09 & & \\
\hline UVM2 & 08:19 & 4400 & $223 \pm 8$ & $5.23 \pm 0.01$ \\
\hline UVM2 & 09:37 & 4400 & $224 \pm 4$ & $5.21 \pm 0.01$ \\
\hline UVM2 & $10: 56$ & 2000 & $225 \pm 3$ & $5.24 \pm 0.01$ \\
\hline$U V M 2$ & $12: 14$ & 4400 & $225 \pm 3$ & $5.25 \pm 0.01$ \\
\hline UVM2 & $13: 32$ & 4400 & $232 \pm 3$ & $5.39 \pm 0.01$ \\
\hline
\end{tabular}

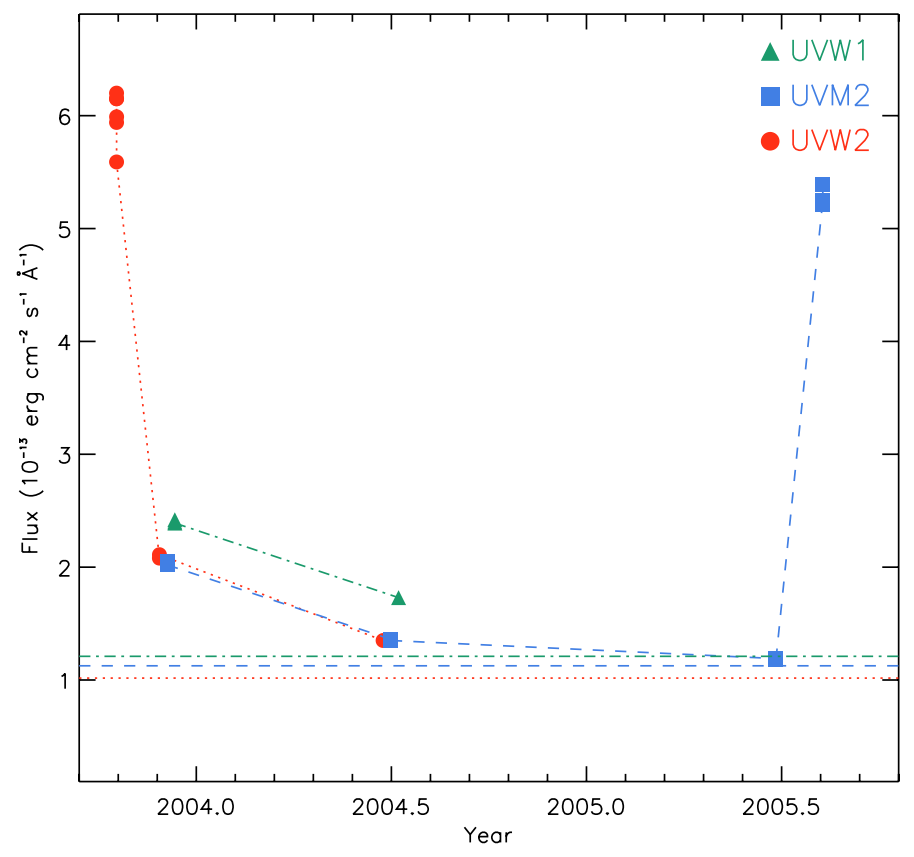

Fig. 3. Optical Monitor UV fluxes during the period October 2003-July 2005. $U V W 1$ (2910 ̊): triangles and dotted-dashed line; UVM2 $(2310 \AA)$ : squares and dashed lines; $U V W 2$ (2120 $\AA$ ): circles and dotted lines. Ordinates are not-dereddened fluxes in units of $10^{-13} \mathrm{erg} \mathrm{cm}^{-2} \mathrm{~s}^{-1} \AA^{-1}$. The bottom lines mark, for reference, the level of the quiescence flux in the three UV bands as derived from IUE data.

On the other hand, if we assume an increasing column density, the shape of the spectrum becomes less sensitive to the blackbody temperature, not being possible to constrain it usefully.

In the third observation (July 2005), obtained near the peak of the outburst, the spectrum is fainter than in November 2003 (see Fig. 2 bottom right). There is no indication of the presence of a supersoft component.

The November 2003 and July 2005 are the first X-ray spectra of AG Dra recorded during an outburst.

The October 2003 XMM observation was taken with the Medium filter in the three EPIC cameras, while the observations of November 2003 and July 2005 were taken with the Thin filter. The October 2003 observation resulted in a negative detection (see Table 1). It is not possible to totally exclude the presence of a soft source, since the filter would have precluded its detection. Nevertheless, given the absence of this component in July 2005 (see below), it is not unreasonable to assume that it is never present close to the visual maximum of the outburst.

The November 2003 observation was obtained when the system had already started the decline from the peak of the outburst. The spectrum shows a soft continuum similar to that observed in quiescence, but weaker, plus an excess emission at energies between 0.5 and $1 \mathrm{keV}$. Again, due to the low countrate, the shape of the spectrum and the limited information at low energies, we cannot distinguish between the different models.

A fit to a blackbody with similar temperature as found in quiescence and absorbed by a similar hydrogen column would give a too low value for the WD radius $\left(0.02 R_{\odot}\right)$. Given the shape of the spectrum, a low temperature $(k T \approx 0.01 \mathrm{keV})$ would require twice as much absorption than in quiescence, and would lead to an unrealistically high luminosity $\left(>10^{6} L_{\odot}\right)$.

\section{The UV data}

XMM-Newton Optical Monitor exposures were performed in parallel with the X-ray observations. The filters used were $U$ (effective wavelength $3440 \AA), U V W 1$ (2910 ̊), UVM2 (2310 ̊) and $U V W 2(2120 \AA)$. Details about the start time and duration of the exposures in each filter are given in Table 2. The $U$ filter was used only in November 2003. The OM instrumental configuration was in all cases such that it allowed, apart form the imaging window, a small window in the centre of the field in "Fast" mode, with a time resolution of $0.5 \mathrm{~s}$. AG Dra was detected in all four OM filters. Due to the brightness of the target, the coincidence loss corrections to the $U V W 1$ filter in October 2003 and to the $U$ filter data of November 2003 are highly uncertain, and therefore these data are not reliable. OM fluxes of AG Dra are summarised in Table 2 and plotted in Fig. 3.

Figure 4 shows the UV light curves of AG Dra during the five observations. For clarity, light curves are plotted with a time binning of $100 \mathrm{~s}$. All the light curves show some degree of variability at different time scales, but without any clear periodicity. The largest variability is observed in the $U V W 2$ data of October 2003, where the flux decreases more than $10 \%$ during the $\sim 7000 \mathrm{~s}$ of observation. Large variations are also present in the $U V W 1$ data of November 2003. 


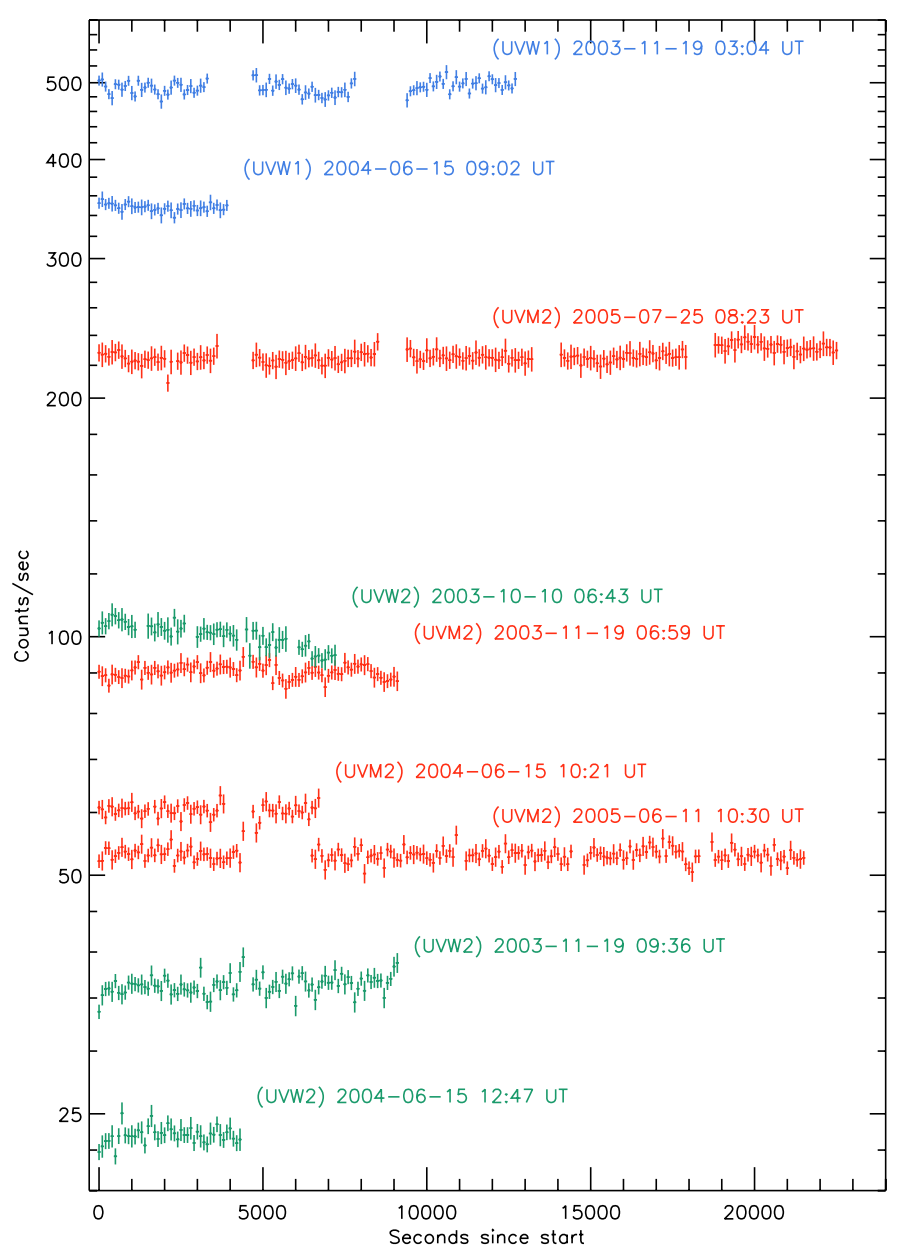

Fig. 4. OM Fast mode light curves (in counts per sec) plotted with a time binning of $100 \mathrm{~s}$. The start time of each observation is indicated. The observations of June 2004 and June 2005 correspond to quiescence epochs, while the other ones correspond to phases of activity. The effective wavelength of the filters are: $2910 \AA$ (UVW1), $2310 \AA$ (UVM2) and $2120 \AA$ (UVW2).

AG Draconis was extensively monitored with the IUE satellite during the period June 1979-May 1995 (González-Riestra et al. 1999). For reference, average IUE fluxes in quiescence are $1.02,1.13$ and $1.21 \times 10^{-13} \mathrm{erg} \mathrm{cm}^{-2} \mathrm{~s}^{-1} \AA^{-1}$ at the effective wavelengths of the $U V W 2, U V M 2$ and $U V W 1$ filters. The difference between the UVM2 fluxes of June 2004 and June 2005 ( $15 \%$ lower in the second observation), at orbital phases 0.36 and 0.00 , respectively, is in good agreement with the orbital modulation detected in IUE data. As discussed among others by González-Riestra et al. (1999), at the wavelengths sampled by all the OM UV filters the dominant contributor to the spectrum is the Balmer recombination continuum. The observed decrease in flux confirms that this emitting region is partially occulted during the orbital motion of the system. During the period covered by the IUE observations, maximum fluxes were reached during outburst in July 1994 for the $U V W 2$ wavelength band $\left(3.61 \times 10^{-12} \mathrm{erg} \mathrm{cm}^{-2} \mathrm{~s}^{-1} \AA^{-1}\right)$, and in March 1985 for UVM2 and $U V W 1$ at 3.28 and $3.32 \times 10^{-13} \mathrm{erg} \mathrm{cm}^{-2} \mathrm{~s}^{-1} \AA^{-1}$, respectively. The XMM ultraviolet fluxes observed during the October 2003 and July 2005 outbursts were slightly higher than those detected with IUE during outbursts (see Table 2 above, and Table 1 in González-Riestra et al. 1999).
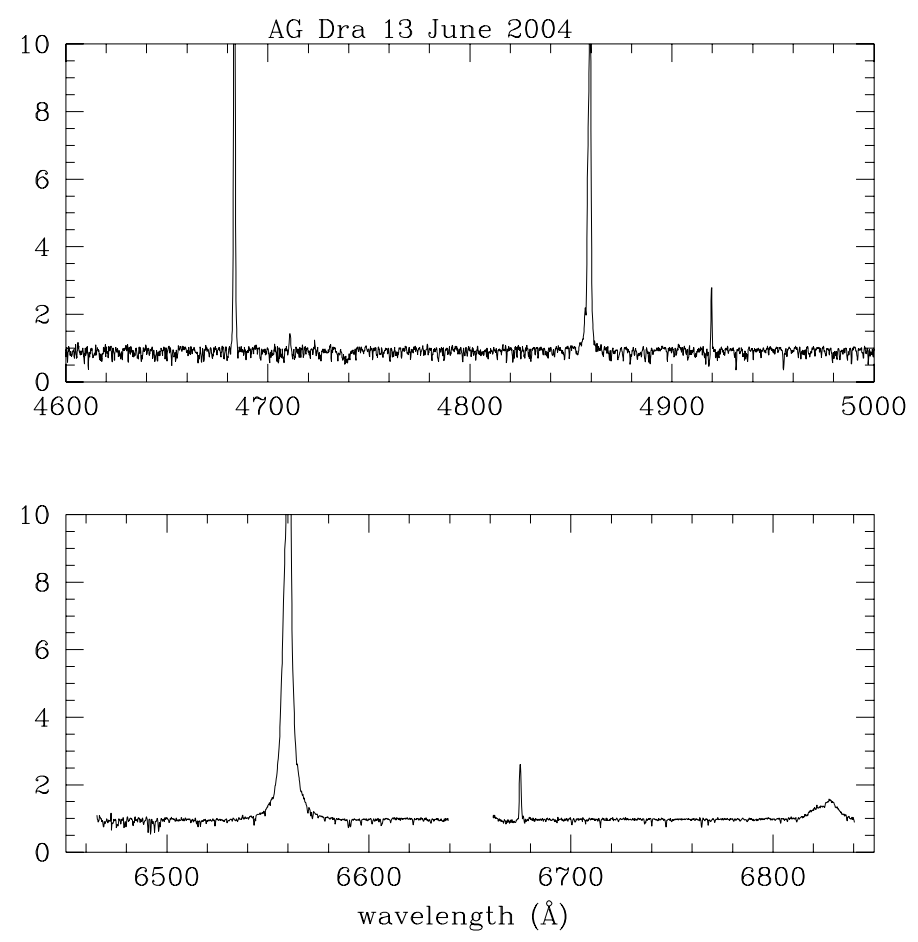

Fig. 5. Portions of the high resolution optical spectrum of AG Dra during the XMM observations of June 2004 (Catania Observatory). Ordinates are fluxes normalised to the continuum.

\section{Optical observations}

In this section we present the results of a campaign of optical observations made in coordination with the XMM observations. Optical spectra of AG Draconis were obtained during 2003-2006 with the Boller and Chivens spectrograph attached to the $122 \mathrm{~cm}$ Galilei telescope of the Asiago Observing Station of the Astronomical Department of the Padova University. The spectra cover the 4000-5200 $\AA$ wavelength range with a resolution of $2 \AA /$ pixel. The star was also observed with the $152 \mathrm{~cm}$ Cassini telescope at the Loiano Observing Station of the Bologna Astronomical Observatory on 5 May and October 6 2003, and on June 14-15 2004. This telescope was equipped with the BFOSC spectrograph, which provided intermediate and echelle spectra covering the 3300-10200 A wavelength range. Echelle spectrograms of AG Dra covering the 4310-6840 A region with a resolution of 0.11 to $0.18 \AA$ per pixel were obtained on June 13-16, 2004 at the M. G. Fracastoro Station (Serra La Nave, Etna) of the Catania Astronomical Observatory. In addition multicolour photometry of AG Dra was collected with the $60 \mathrm{~cm}$ telescopes at the Skalnaté Pleso and Stara Lesna observatories (see Skopal et al. 2004 for details), and with the Greve $30 \mathrm{~cm}$ telescope (see Montagni et al. 1996).

Figure 5 shows the high resolution spectrum of AG Dra taken in June 2004 near the time of the XMM observations. The spectrum is typical of the star being in quiescence, with the continuum containing the photospheric absorption lines of the red giant star. The corresponding heliocentric radial velocity is $-144.1 \pm 0.2 \mathrm{~km} \mathrm{~s}^{-1}$, in agreement with the value expected at that orbital phase according to the adopted ephemeris in Sect. 1. Many emission lines are present: $\mathrm{H} \alpha$, very strong with an equivalent width of $88 \AA$, the He II $4686 \AA$ emission line $\left(W_{\text {eq }}=18 \AA\right.$ ), 

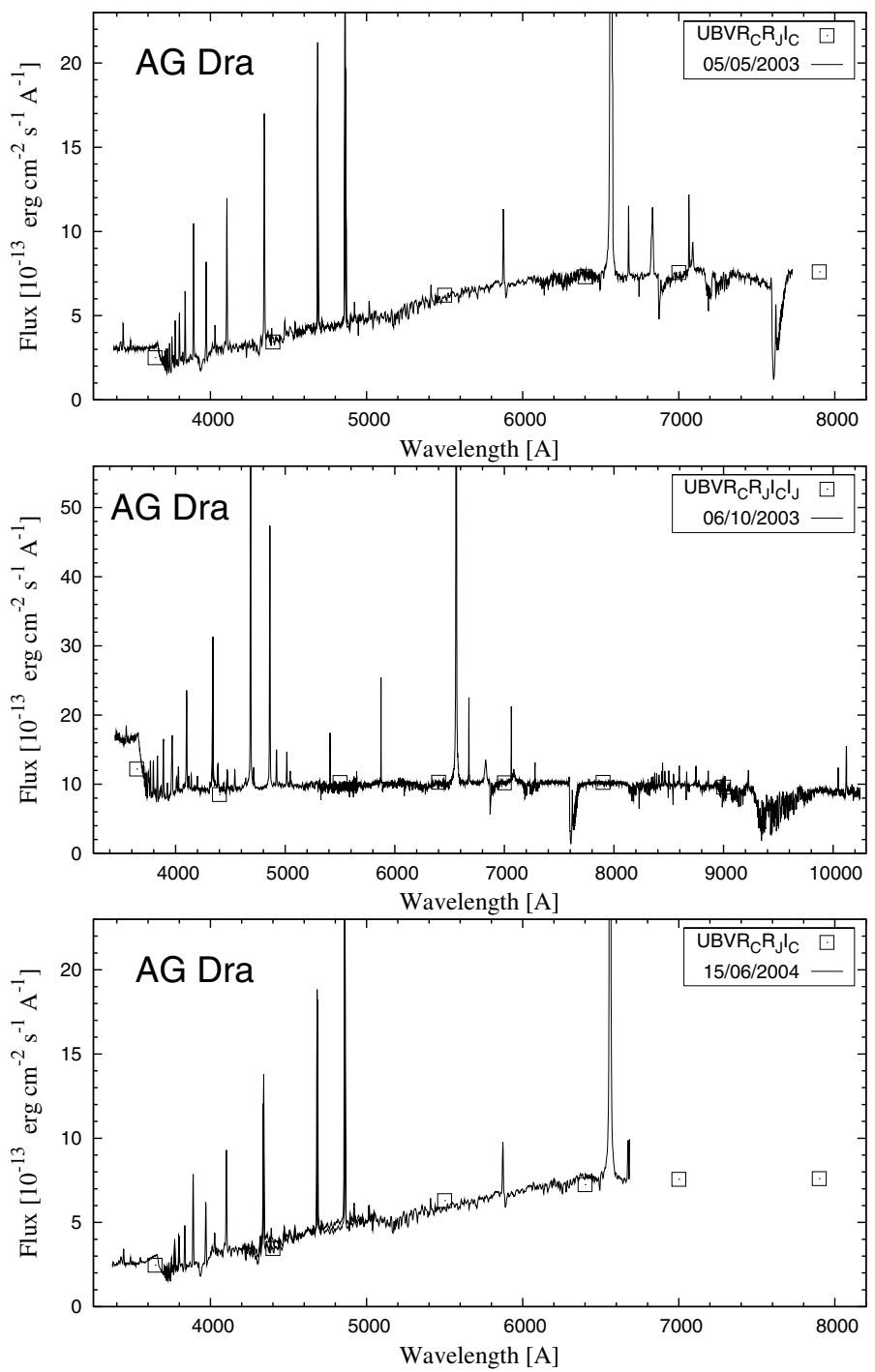

Fig. 6. The Loiano Observatory spectrum of AG Dra during quiescence (May 2003 and June 2004, top and bottom panels) and outburst (October 2003, middle panel with a wider wavelength range). The rednear IR part of October 2003 is an echelle spectrum. Ordinates are dereddened fluxes (see text for details). Open squares indicate the photometric data.

many He I lines (He I $5876 \AA, W_{\text {eq }}=2.4 \AA$ ), and the Raman scattering line at $6830 \AA\left(W_{\text {eq }}=\sim 6.6 \AA\right)$.

Figure 6 shows the Loiano Observatory spectra of AG Dra obtained before and during the 2003 outburst, and at the time of the XMM observations in June 2004. The spectra were calibrated with the aid of the simultaneous multicolour photometry. The measured photometric flux points were corrected for the influence of emission lines. Uncertainties of the continuum calibration are of a few percent for the star's brightness around 9 mag in the $V-R$ region (see Skopal 2007). Observations were dereddened with $E_{B-V}=0.08$. All the spectra show the Balmer jump in emission, indicating the presence of strong hydrogen recombination emission (see Fig. 6). The October 2003 outburst was marked by a large increase of the emission line and continuum flux. The energy distribution is flatter in the optical range due to the increased nebular emission. This emission excess partially masks the red-giant photospheric lines. For instance, a close inspection of the spectrum shows a significant weakening of the Ca II absorption at $3933 \AA$ and of the $g$-band at $4308 \AA$.

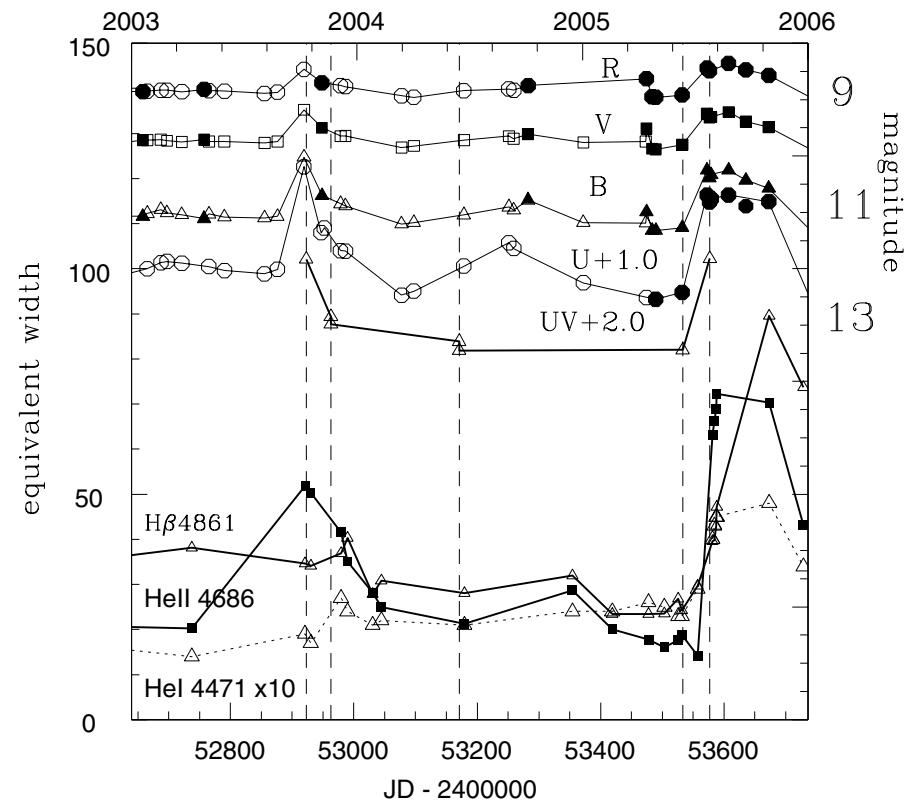

Fig. 7. Equivalent width variation of $\mathrm{H} \beta$ (open circles), He II $4686 \AA$ (filled squares) and He I $4471 \AA$ (open triangles) during 2003-2006. The $U V U B V R$ light curves are shown at the top of the figure, with vertical offsets for $U$ and UV. Filled and open symbols for the optical photometry refer to Greve and Skalnate Pleso observations, respectively. The UV magnitudes are derived from the $U V W 2$ and $U V M 2$ OM fluxes (see text). The vertical lines mark the times of the XMM observations.

We have measured the equivalent width of the emission lines in our optical spectra of AG Dra collected during 2003-2006, and compared them with the optical and ultraviolet photometric curves. Some results are illustrated in Fig. 7. The $\Delta R$ magnitudes collected at Skalnaté Pleso were converted into Cousins $R$ magnitudes assuming a correcting term of $+9.53 \pm 0.03$, as derived from a preliminary comparison with the Greve $R$ magnitudes collected at close dates. The figure also reports the XMM ultraviolet magnitudes observed with the $U V M 2$ and $U V W 2$ filters ${ }^{1}$.

The photometric observations show that during the October 2003 outburst AG Dra brightened by about 0.8 in the visual up to about 1.8 in the $U$-band (see also Skopal et al. 2004). The ultraviolet XMM observations show that near the 2003 light maximum AG Dra was about 1.6 brighter in the far-UV than during quiescence. The same amplitude of variation in the visual and in the ultraviolet was displayed by the following outburst of July 2005, but the duration of the light maximum was longer. The interoutburst period was characterised by an ample wavelike variation of the $U$ band that reached its maximum at the orbital phase 0.50 (beginning of September 2004).

During the October 2003 outburst the optical spectrum of AG Dra was characterised by a large increase of the He II $4686 \AA$ emission line, while the flux of the hydrogen and neutral helium emission lines varied by an amount similar to that of the underlying continuum, as indicated by the small change in their equivalent widths. The November 2003 XMM-Newton observations were made during fading after the outburst when the star luminosity had decreased from the maximum by 1 . 2 in $U$ and by $0^{\mathrm{m}} .4$ in $V$. At that time the He II $4686 \AA$ emission line had

\footnotetext{
1 The UV fluxes were converted into magnitudes assuming $m(\mathrm{UV})+$ $2.5 \times \log$ (flux) $=-20.88$ and -20.72 for $U V M 2$ and $U V W 2$, respectively. In the figure, data from both bands are reported, since the difference for
} common dates is small. 


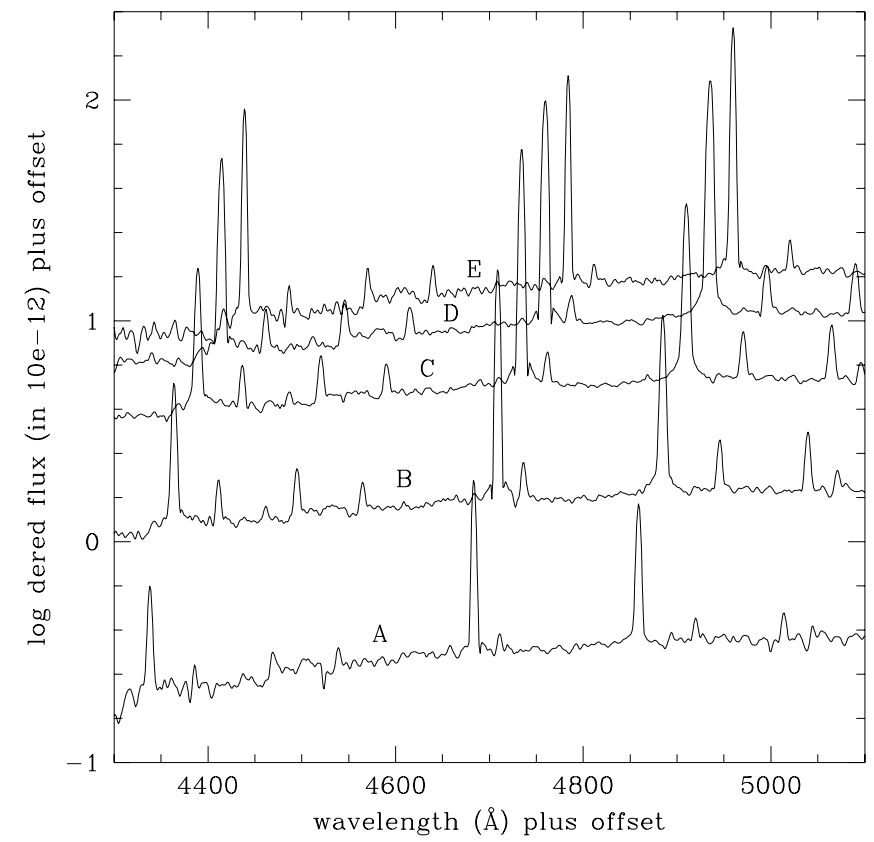

Fig. 8. The spectral variations of AG Dra during the 2005 outburst. Ordinates are the logarithm of dereddened fluxes. Vertical (+0.4 in $\log$ ) and horizontal $(+25 \AA)$ offsets have been applied to successive spectra to avoid overlapping. The observation dates are, from bottom to top: A: July 5 (before outburst), B: July 29, C: August 4 and August 5 (red), D: October 29, E: December 24. Spectra collected with the Asiago $122 \mathrm{~cm}$ telescope. The three most prominent emission lines are $\mathrm{H} \gamma$, He II $4686 \AA$, and $\mathrm{H} \beta$.

strongly weakened, but it was still stronger than $\mathrm{H} \beta$. At the time of the June 2004 XMM observations AG Dra had recovered its quiescent phase.

The July 2005 was marked by a dramatic increase (from $14 \AA$ to $70 \AA$ ) of the equivalent width of the He II $4686 \AA$ emission line. Figure 8 shows the evolution of the blue spectrum of AG Dra between July and December 2005. In the figure the spectra are plotted on a logarithmic scale to better illustrate the continuum and emission line variation.

\section{Discussion}

The luminosity of the WD component of AG Dra during quiescence is very high, of the same order as that of the K-giant companion (e.g. Greiner et al. 1997). This high luminosity is explained by stationary thermonuclear burning on the WD surface of hydrogen rich matter accreted from the K-giant wind at a high rate (around $3 \times 10^{-8} M_{\odot} \mathrm{yr}^{-1}$, for a distance of $2.5 \mathrm{kpc}$, Greiner et al. 1997), with a fairly low temperature of the emitting region, around $10^{5} \mathrm{~K}$, so that most of the radiation is emitted in the EUV frequency range.

We have found a significant difference between the X-ray spectra of AG Dra of June 2004 and June 2005. While both spectra have a similar shape in the $0.2-0.4 \mathrm{keV}$ energy range, the countrate is $20 \%$ lower in June 2005. Since this latter observation was made near conjunction of the system with the red giant in front of the WD companion (orbital phase 0.01), the flux decrease could be associated with a partial occultation of the WD surface. ROSAT observations have detected orbital variability of the X-ray flux. Greiner et al. (1997) reported a 20\% decrease in the ROSAT countrate in May 1993 (JD 2449119), slightly before the time of the minimum of the $U$-band light

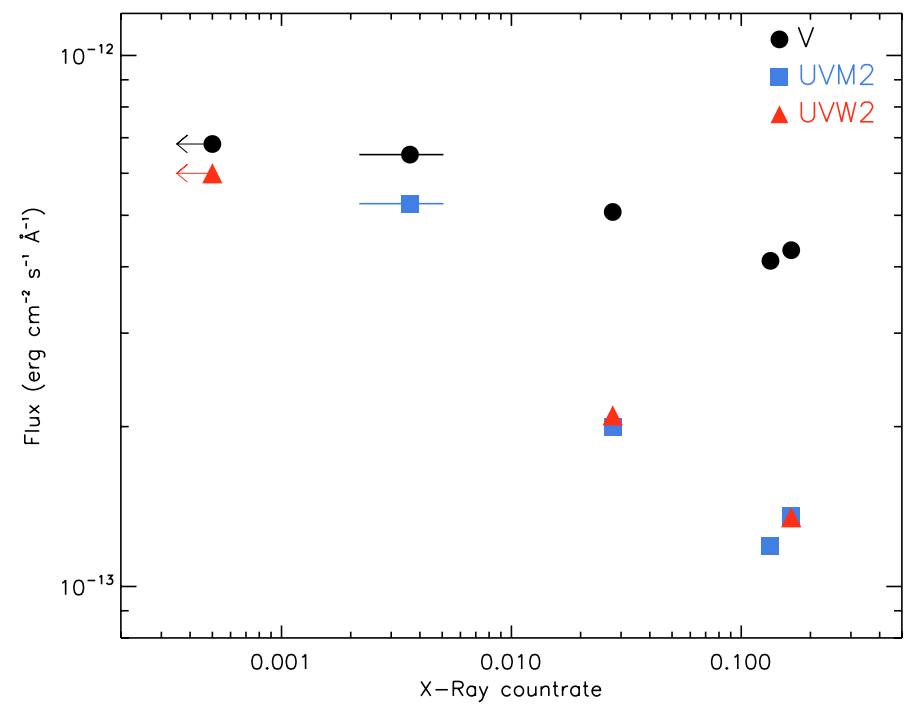

Fig. 9. Anticorrelation between the X-ray fluxes (abscissae) and the visual (circles) and UV brightness (UVM2: squares, $U V W 2$ : triangles). Abscissae: EPIC-pn countrates. Ordinates: visual and ultraviolet fluxes. For October 2003 the X-ray upper limit is given. This observation was performed using the Medium filter and therefore the countrate is not directly comparable with the data of the other epochs.

curve at orbital phase 0.99 (see top centre of Fig. 1). They also noted that the X-ray flux decrease occurred very slowly over a time interval of two months, suggesting a total duration of the minimum of at least 4 months, if the modulation was symmetric in shape. This time interval is too long if due to the eclipse of the WD by the red giant companion.

The occultation hypothesis is also in disagreement with the past ultraviolet observations. In fact, the IUE data described in González-Riestra et al. (1999) show the existence during quiescence of a marked orbital modulation of the UV continuum in phase with the $U$-band light curve, whose amplitude decreases at the shortest ultraviolet wavelengths. Since, as discussed by González-Riestra et al. (1999), during quiescence the WD radiation dominates the IUE short wavelength limit; this indicates that the WD component is not occulted, probably because of the shallow inclination of the orbit. The $\sim 15 \%$ flux difference found in the XMM-UVM2 filter between June 2004 and June 2005 noted above has to be attributed the orbital modulation of the nebular emission which still dominates the energy spectrum at the filter wavelength of $2310 \AA$ (see Skopal 2005).

An alternative hypothesis suggested by Greiner et al. (1997) to explain the X-ray minimum in May 1993 was the presence of an unreported minor light maximum in the $U, B$ and $V$ bands that occurred on JD 2449210 after that X-ray minimum. We notice that the AAVSO light curve also gives a visual maximum of about 9.7 around JD 2449200-220. The XMM observations of June 2005 were made about one month before the start of the new outburst. Hence also in this case the X-ray flux decrease could be associated with the successive visual brightening. We cannot yet decide whether there is an association with an X-ray decrease anticipating the visual brightening, or if the June 2005 result can be interpreted as an accidental fluctuation of the X-ray flux during one orbit, or from one orbit to another. This would require further X-ray observations with short-time spacing (a few weeks).

During all the five outbursts so far observed with X-ray satellites (EXOSAT, ROSAT and XMM), the soft X-rays weakened 
independently of the outburst's peak luminosity. As discussed in Sect. 4, during the October 2003 and July 2005 outbursts covered by XMM observations the optical spectrum of AG Dra was characterised in the first phases of the outburst by a large increase of the He II $4686 \AA$ emission line. In particular, during the July 2005 luminosity rise that was well monitored spectroscopically, the brightening of the He II line was very rapid, while the rise of $\mathrm{H} \beta$ was slower, or, in the case of the 2003 outburst, $\mathrm{H} \beta$ varied by an amount similar to that of the underlying continuum. These results indicate that the flux of photons beyond the ionisation limit of $\mathrm{He}^{+}$greatly increased during the first stages of the outburst, with a smaller increase of the photon flux near the neutral helium and hydrogen ionisation limits. We suggest that the first stages of both outbursts were characterised by an increase of the hot source temperature at constant radius. This phase was followed by the expansion of the WD radius and decrease of its surface temperature, as indicated by the decrease of the $4686 / \mathrm{H} \beta$ ratio after the 2003 and 2005 light maxima.

The mechanism producing the outbursts in AG Dra is still unknown. The recurrence time of about one year of individual outbursts during an active phase might suggest an association with the second $\sim 355$ day variability cycle of AG Dra, which is sometimes attributed to pulsation of the K-star (e.g. Galis et al. 1999; Friedjung et al. 2003). However, according to the $355 \mathrm{~d}$ cycle, the phase at the onset of the outburst is different in different activity epochs (0.8-0.9 during 1994-1998 and 0.3-0.4 in the more recent ones).

According to González-Riestra et al. (1999) the decrease of the X-ray flux during outburst can be attributed to two mechanisms depending on the outburst type. During the weaker 1985 and 1986 outbursts, the WD is surrounded by a hot envelope with a large EUV brightness, but opaque to the soft X-rays due to the presence of a hot wind with a large opacity shortward of the $N^{+4}$ ionisation limit at $127 \AA$ (hot outbursts), similar to the case of SMC 3 discussed by Jordan et al. (1996), in which case the main absorber would be ionised carbon. During the strong 1994 and 1995 outbursts the envelope extended to several stellar radii, with a lower effective temperature and EUV brightness (cool outbursts). However, we cannot exclude that during the earlier phases of the stronger outbursts AG Dra passed through a hot phase, as suggested by the IUE observations of the rising phase of the 1980 and 1994 outbursts. This seems to be the case for the 2003 and 2005 outbursts, where, as discussed above, the initial temperature rise is marked by the increase of the $\mathrm{He}$ II $\lambda 4686 / \mathrm{H} \beta$ ratio. This phase was followed after the light maximum by an increase of the hydrogen recombination lines and of a marked change in the $E U V$ colour temperature.

An increase in the temperature of the hot source also has been observed in the first stages of the 1997 and 2000 outbursts of $Z$ And (Sokoloski et al. 2006). Both systems present other similarities, for instance the appearance of outbursts in pairs or in groups of decreasing peak intensity. Sokoloski et al. (2006) have proposed the "combination nova" model to explain the outbursts of $Z$ And. In this scenario, outbursts are caused by a disk instability that, in some cases, triggers an increase in the nuclear burning on the hot star surface. There are however some important differences that lead us to think that this mechanism is not working in the case of AG Dra. First, there is no evidence so far for the existence of an accretion disk in AG Dra (e.g. flickering, as in $Z$ And). Second, the behaviour of the X-ray emission is opposite in both systems. While $Z$ And is a weak X-ray source in quiescence and its X-ray flux increases by a factor of ten during outburst, AG Dra shows a marked anticorrelation between optical and X-ray luminosity.

The June 2005 XMM observations suggest the presence of a $0.5-0.6 \mathrm{keV}$ flux excess with respect to the soft blackbody spectrum. This excess also seems to be present during the outburst observations of November 2003 and July 2005. A similar excess was also noted by Anderson et al. (1981) in the analysis of the Einstein Observatory observations during quiescence in 1980. If the presence of this excess during both quiescence and outburst is confirmed, we conclude that it originates far from the WD surface, probably in a diffuse shocked region possibly associated with the knots observed at radio wavelengths (see Mikolajewska 2002; Ogley et al. 2002). It could originate from the interaction of the WD wind and of matter ejected during the outbursts with circumbinary matter fed by the red giant's wind.

\section{Conclusions}

We have analysed X-ray and UV data of AG Dra taken with XMM-Newton at five different epochs: two at quiescence (June 2004 and June 2005), and three in active phases (October 2003, November 2003 and July 2005).

The quiescence X-ray data show a strong soft component, similar to that seen in the ROSAT-PSPC observations. The very soft shape of the spectrum precludes an accurate derivation of the spectral parameters, but it is consistent with a blackbody spectrum of $\sim 170000 \mathrm{~K}$, affected by an interstellar column density of $\sim 2.7 \times 10^{20} \mathrm{~cm}^{-2}$, slightly below the galactic value. The estimated radius for the WD is about $0.06 R_{\odot}$. The derived luminosity of about $2600 L_{\odot}$ is a strong indication of the existence of a stable layer of fresh accreted hydrogen burning on the surface of the WD.

During the luminosity variations X-ray flux is anti-correlated with the optical/UV emission. This confirms the behaviour previously observed with EXOSAT and ROSAT, and appears to be a general feature, independent of the type and strength of the outburst. The small flux changes observed with XMM in June 2005 and with ROSAT in May 1993 are probably precursors of the subsequent light maxima, rather than orbital effects.

We have found that both events of October 2003 and July 2005 were hot outbursts, characterised by a large increase of the He II recombination lines. After the light maxima the hot source temperature decreased. The three XMM observations in outburst were taken at different activity levels. In October 2003 and July 2005 the system was very close to the peak of the outburst, while in November 2003 it was already declining. The non-detection in the first observation could in principle be ascribed to the use of the EPIC Medium filter that suppressed the soft X-ray emission. However, in July 2005 the system was at approximately the same optical brightness and the X-ray emission was extremely faint, with no trace of soft flux, even through the EPIC Thin filter. We therefore conclude that most likely the X-ray soft component was not present in October 2003 either. However, forty days later it had reappeared. X-ray observations at the earliest (and probably hottest) phases of the outburst are required to monitor the change of the WD surface as the result of the explosion, and to understand the origin of the explosion itself. Chance X-ray observations before the beginning of the optical outburst would be even more important. Unfortunately, due to the limited spectral range available, we cannot ascribe the decrease in the soft X-ray flux during outburst to either of the two possible phenomena: decrease of the WD temperature, or increase of the envelope opacity. In this regard the variable hot wind of the WD might play a crucial role. 
The UV flux closely follows the optical behaviour. High temporal resolution observations show some degree of variability at different time scales, but without any clear periodicity.

Finally, there is some evidence for a high energy X-ray component, which is probably present both in quiescence and during outburst. Its putative association with the circumstellar nebulae observed at radio wavelengths is a point that will require further investigation.

Acknowledgements. This work is based on observations obtained with XMM-Newton, an ESA science mission with instruments and contributions directly funded by ESA Member States and NASA. It is also based on optical spectroscopic observations obtained at the Asiago, Loiano and Catania Observatories, and on archive IUE data. We thanks V. F. Polcaro for collecting some spectra of AG Dra at the Loiano telescope. We are grateful to M. Friedjung for his comments that have greatly improved this paper. We acknowledge with thanks the variable star observations from the AAVSO International Database contributed by observers worldwide and used in this research. This research was in part supported by a grant of the Slovak Academy of Sciences No. 2/7010/27.

\section{References}

Anderson, C. M., Cassinelli, J. P., \& Sanders, W. T. 1981, ApJ, 247, L127

Bastian, U. 1998, A\&A, 329, L61

Friedjung, M., Gális, R., Hric, L., \& Petrík, K. 2003, A\&A, 400, 595

Gális, R., Hric, L., Friedjung, M., \& Petrík, K. 1999, A\&A, 348, 533
González-Riestra, R., Viotti, R., Iijima, T., \& Greiner, J. 1999, A\&A, 347, 478 Greiner, J., Bickert, K., Luthard, R., et al. 1997, A\&A, 322, 576

Jansen, F., Lumb, D., Altieri, B., et al. 2001, A\&A, 365, L1

Jordan, S., Schmutz, W., Wolff, B., et al. 1996, A\&A, 312, 897

Kenyon, S. J. 1988, in The Symbiotic Phenomenon, ed. J. Mikolajewska, et al., IAU Coll., 103, ASSL, 145, 11

Meinunger, L. 1979, IBVS, 1611

Mikolajewska, J. 2002, MNRAS, 335, L33

Mikolajewska, J., Kenyon, S. J., Mikolajewski, M., et al. 1995, AJ, 109, 1289

Mukai, K., Ishida, M., Kilbourne, C., et al. 2007, PASJ, 59, 177

Mürset, U., Wolff, B., \& Jordan, S. 1997, A\&A, 319, 201

Montagni, F., Maesano, M., Viotti, R., et al. 1996, IBVS, 4336

Ogley, R. N., Chaty, S., Crocker, M., et al. 2002, MNRAS, 330, 772

Piro, L., Cassatella, A., Spinoglio, L., Viotti, R., \& Altamore, A. 1985, IAU Circ., 4082

Skopal, A., Pribulla, T., Vanko, M., et al. 2004, Contrib. Astron. Obs. Skalnaté Pleso, 34, 1

Skopal, A. 2005, A\&A, 440, 995

Skopal, A. 2007, New Astron., 12, 597

Sokoloski, J. L., Kenyon, S. J., Espey, B. R., et al. 2006, ApJ, 636, 1002

Viotti, R., Ricciardi, O., Ponz, D., et al. 1983, A\&A, 119, 285

Viotti, R., Altamore, A., Baratta, G. B., Cassatella, A., \& Friedjung, M. 1984, ApJ, 283, 226

Viotti, R., Giommi, P., Friedjung, M., \& Altamore, A. 1995, in Cataclysmic Variables, ed. A. Bianchini, M. Della Valle, \& M. Orio, ASSL, 205, 195

Viotti, R. F., Montagni, F., Maesano, M., et al. 2003, in Symbiotic Stars Probing Stellar Evolution, ed. R. L. M. Corradi, J. Mikolajewska, \& T. J. Mahoney, ASP Conf. Ser., 303, 298 